\title{
Selection of reliable reference genes for quantitative real-time PCR in human T cells and neutrophils
}

\author{
Carola Ledderose ${ }^{1,2}$, Jens Heyn ${ }^{2}$, Elisabeth Limbeck ${ }^{2}$ and Simone Kreth ${ }^{2 *}$
}

\begin{abstract}
Background: The choice of reliable reference genes is a prerequisite for valid results when analyzing gene expression with real-time quantitative PCR (qPCR). This method is frequently applied to study gene expression patterns in immune cells, yet a thorough validation of potential reference genes is still lacking for most leukocyte subtypes and most models of their in vitro stimulation. In the current study, we evaluated the expression stability of common reference genes in two widely used cell culture models-anti-CD3/CD28 activated T cells and lipopolysaccharide stimulated neutrophils-as well as in unselected untreated leukocytes.

Results: The mRNA expression of 17 (T cells), 7 (neutrophils) or 8 (unselected leukocytes) potential reference genes was quantified by reverse transcription $\mathrm{gPCR}$, and a ranking of the preselected candidate genes according to their expression stability was calculated using the programs NormFinder, geNorm and BestKeeper. IPO8, RPL13A, TBP and SDHA were identified as suitable reference genes in T cells. TBP, ACTB and SDHA were stably expressed in neutrophils. TBP and SDHA were also the most stable genes in untreated total blood leukocytes. The critical impact of reference gene selection on the estimated target gene expression is demonstrated for $\mathrm{L}-2$ and $\mathrm{FlH}$ expression in T cells.

Conclusions: The study provides a shortlist of suitable reference genes for normalization of gene expression data in unstimulated and stimulated T cells, unstimulated and stimulated neutrophils and in unselected leukocytes.
\end{abstract}

\section{Background}

Due to its high sensitivity, specificity and resolution, quantitative real-time PCR (qPCR) has become the method of choice for gene expression analyses of selected genes [1-3]. However, reverse transcription (RT) qPCR measurements are influenced by a variety of unspecific factors, including the amount and quality of the isolated RNA and efficiencies of reverse transcription and PCR amplification, which makes accurate normalization a prerequisite for reliable results [1,4-6]. The most commonly applied normalization strategy involves the use of reference genes as internal controls, whose expression should be constant in all samples under investigation [7]. Since it has become clear, though, that conventional reference genes, such as glyceraldehyde-3-

\footnotetext{
* Correspondence: simone.kreth@med.uni-muenchen.de

${ }^{2}$ Department of Anesthesiology, Ludwig-Maximilians-University Munich, Germany

Full list of author information is available at the end of the article
}

phosphate dehydrogenase $(G A P D H)$ or $\beta$-actin $(A C T B)$, are regulated under certain circumstances leading to invalid results $[7,8]$, it is essential to validate the suitability of potential reference genes for the specific experimental conditions.

The study of gene expression patterns in immune cells is a promising approach to gain insight into complex regulatory mechanisms associated with immunemediated disease [9]. Although RT-qPCR is frequently employed for gene expression analysis in leukocytes, a thorough validation of reference gene stability has not been described yet. Data are not only missing for the appropriate normalization of mRNA levels in unselected leukocytes, but are also scarce with respect to leukocyte subtypes or activation procedures [10-12]. Stimulating T cells with anti-CD3/CD28 beads to mimic the activation by antigen-presenting cells [13], for example, or treating neutrophils with lipopolysaccharide (LPS) [14-16] are two well-established in vitro models in the investigation
C Biomed Central

() 2011 Kreth et al; licensee BioMed Central Ltd. This is an open access article distributed under the terms of the Creative Commons Attribution License (http://creativecommons.org/licenses/by/2.0), which permits unrestricted use, distribution, and reproduction in any medium, provided the original work is properly cited. 
of inflammatory, infectious or autoimmune disease; a systematic validation of reference gene stability has thus far been lacking for either model, though.

In the present study we investigated the expression stability of potential reference genes in unstimulated and anti-CD3/CD28 activated T cells and in unstimulated and LPS-stimulated neutrophils, using the three software applications geNorm [6], NormFinder [4] and BestKeeper [5]. Based on these results, we further identified reference genes that can be used as universal normalizers in gene expression studies in unselected leukocyte populations. Furthermore, we show that the use of unstable reference genes is prone to cause highly misleading results, which underlines the importance of a thorough selection and evaluation of reference genes for RT-qPCR experiments in immune cells.

\section{Methods \\ Isolation and stimulation of T lymphocytes and neutrophils}

Blood withdrawal from healthy volunteers was approved by the institutional ethics committee of the Ludwig Maximilians University, Munich, Germany, and written informed consent was obtained. $T$ cells were isolated from peripheral blood mononuclear cells by negative selection using the Pan $\mathrm{T}$ cell isolation kit II (Miltenyi Biotec) according to the manufacturer's instructions. Neutrophils were separated from whole blood by continous percoll gradient density centrifugation as previously described [17]. Cells were cultured in RPMI1640 medium (Sigma-Aldrich) supplemented with 10\% heat-inactivated fetal calf serum (Biochrom) and L-glutamine $(\mathrm{Gibco})$ at $37^{\circ} \mathrm{C}$ in $5 \% \mathrm{CO}_{2}$. T cells $\left(1 \times 10^{6} / \mathrm{ml}\right)$ were stimulated with anti-CD3/CD28 beads (Invitrogen) at a bead-to-cell ratio of $1: 1$ and harvested after 24 hours. Neutrophils $\left(1.5 \times 10^{6} / \mathrm{ml}\right)$ were stimulated for 6 hours with $100 \mathrm{ng} / \mathrm{ml}$ LPS (E.O55.B5, Sigma-Aldrich).

\section{RNA extraction and cDNA synthesis}

Total RNA was isolated using the RNAqueous Kit (Ambion) followed by DNase treatment (TurboDNase, Ambion) according to the manufacturer's instructions. Total blood leukocyte RNA was extracted from $10 \mathrm{ml}$ whole blood by use of the LeukoLOCK system (Ambion) following the suggested protocol. RNA quantity and purity were measured with a NanoDrop 2000 spectrophotometer (Thermo Scientific), and only samples with $\mathrm{A}_{260} / \mathrm{A}_{280}$ ratios between 1.80 and 2.00 were analyzed further. The integrity of RNA samples was confirmed by electrophoresis on a $1 \%$ agarose gel. Firststrand cDNA was synthesized from equal amounts of RNA (1000 ng) using Superscript III reverse transcriptase (Invitrogen) and random hexamers and oligo(dT) primers as described [17].

\section{Quantitative real-time PCR}

17 commonly used reference genes were selected as candidate genes (Table 1). Real-time PCR was performed in duplicate on a LightCycler ${ }^{\circledR} 480$ instrument (Roche Diagnostics) using equal amounts (10 ng) of reverse transcribed total RNA and pre-validated probebased RealTime ready ${ }^{\circledR}$ assays (Roche Diagnostics; see Additional file 1 Table S1 for Assay ID and amplicon location). Interleukin-2 (IL-2) and factor inhibiting hypoxia inducible factor $(\mathrm{FIH})$ were chosen as exemplary target genes, using the following primers and Universal ProbeLibrary (UPL) probes (Roche Diagnostics): IL-2: 5' AAGTTTTACATGCCCAAGAAGG 3' (forward primer), 5' AAGTGAAAGTTTTTGCTTTGAGCTA 3' (reverse primer), UPL probe \#65; FIH: 5' ACCCT GTTCATCACCCATGT 3' (forward primer), 5' TCTCG TAGTCGGGATTGTCA 3' (reverse primer), UPL probe $\# 21$. With the exception of $18 \mathrm{~S}$, all assays were designed to span at least one intron. Negative controls without the addition of cDNA were included to verify the absence of contamination. To avoid inter-run variation, the same gene was tested in the same run on different samples [6]. The cycling conditions comprised an inital denaturation phase at $95^{\circ} \mathrm{C}$ for $5 \mathrm{~min}$, followed by 45 amplification cycles at $95^{\circ} \mathrm{C}$ for $10 \mathrm{~s}, 60^{\circ} \mathrm{C}$ for $30 \mathrm{~s}$ and $72^{\circ} \mathrm{C}$ for $15 \mathrm{~s}$. Quantification cycle $\left(\mathrm{C}_{\mathrm{q}}\right)$ values were calculated employing the "second derivative maximum" method as computed by the LightCycler software. Amplification efficiencies were determined for all qPCR assays by calculating calibration curves from 5- to 10fold serial dilutions from pooled cDNA using the equation $E=10^{[-1 / \text { slope }]}$. Efficiencies ranged from $89.2 \%$ $(A L A S)$ to $107.5 \%(A C T B)$ with $r^{2}>1.98$ (see Table S1 for $E$ and $\mathrm{r}^{2}$ values for each assay).

\section{Statistical data analysis}

The Kolmogorov-Smirnov test was applied to determine whether the distribution of the differences between $\mathrm{C}_{\mathrm{q}}$ values of paired samples deviated from a normal distribution. Intergroup comparisons were performed by paired t-test or Wilcoxon signed rank test, if data were normally or not normally distributed, respectively, and candidate genes showing differential expression ( $\mathrm{p}<$ $0.05)$ were ruled out from further analyses. Expression stability of potential reference genes was evaluated by applying three generally accepted [1] Excel-based software tools-BestKeeper [5], geNorm [6] and NormFinder [4]-according to the instructions provided by the developers. The BestKeeper software suggests a preliminary ranking of candidate reference genes based on $\mathrm{C}_{\mathrm{q}}$ variation in expression. Furthermore, it estimates the expression stability by performing a pair-wise correlation analysis for each pair of candidate genes. The program geNorm provides a measure of gene expression stability 
Table 1 Candidate reference genes evaluated in this study.

\begin{tabular}{|c|c|c|c|}
\hline Symbol & Name & Function & Accession No. ${ }^{\mathrm{a}}$ \\
\hline ACTB & $\beta$-actin & cytoskeletal structural protein & NM_001101 \\
\hline ALAS 1 & 5-aminolevulinate synthase 1 & heme biosynthetic pathway & $\begin{array}{l}\text { NM_000688 } \\
\text { NM_199166 }\end{array}$ \\
\hline B2M & $\beta$-2-microglobulin & $\beta$-chain of MHC I molecules & NM_004048 \\
\hline GAPDH & glyceraldehyde-3-phosphate dehydro-genase & carbohydrate metabolism & NM_002046 \\
\hline $\mathrm{HBB}$ & $\beta$-hemoglobin & hemoglobin $\beta$-chain & NM_00518 \\
\hline HMBS & hydroxymethyl-bilane synthase & heme biosynthetic pathway & $\begin{array}{l}\text { NM_000190 } \\
\text { NM_001024382 }\end{array}$ \\
\hline HPRT1 & hypoxanthine phosphoribosyl-transferase 1 & purine salvage pathway & NM_000194 \\
\hline IPO8 & importin-8 & nuclear import of proteins & $\begin{array}{l}\text { NM_001190995 } \\
\text { NM_006390 }\end{array}$ \\
\hline PGK1 & phosphoglycerate kinase 1 & glycolysis & NM_000291 \\
\hline PPIA & peptidylprolyl isomerase A & protein folding & NM_021130 \\
\hline RPLPO & ribosomal protein, large, P0 & ribosomal protein, translation & $\begin{array}{l}\text { NM_001002 } \\
\text { NM_053275 }\end{array}$ \\
\hline RPL13A & ribosomal protein L13A & ribosomal protein, translation & $\begin{array}{l}\text { NM_012423 } \\
\text { NR_026712 }\end{array}$ \\
\hline SDHA & succinate dehydrogenase complex, subunit A & mitochondrial respiratory chain & NM_004168 \\
\hline TBP & TATA box binding protein & general RNA polymerase II transcription factor & NM_003194 \\
\hline TFRC & transferrin receptor $(\mathrm{p} 90, \mathrm{CD} 71)$ & cellular iron homeostasis & $\begin{array}{l}\text { NM_001128148 } \\
\text { NM_003234 }\end{array}$ \\
\hline YWHAZ & $\begin{array}{l}\text { tyrosine-3-monooxygenase/tryptophan 5-monooxygenase activation } \\
\text { protein, zeta polypeptide }\end{array}$ & $\begin{array}{l}\text { binding to phosphorylated serine residues, } \\
\text { signal transduction }\end{array}$ & $\begin{array}{l}\text { NM_001135699 } \\
\text { NM_001135700 } \\
\text { NM_001135701 } \\
\text { NM_001135702 } \\
\text { NM_003406 } \\
\text { NM_145690 }\end{array}$ \\
\hline $18 \mathrm{~S}$ & RNA, 18 s ribosomal 1 & ribosomal RNA, translation & NR_003286 \\
\hline
\end{tabular}

${ }^{a}$ NCBI Reference Sequence database http://www.ncbi.nlm.nih.gov/RefSeq/

$(M)$ by calculating the average pairwise variation of each control gene from all the other control gene candidates. In addition, it performs a ranking of the candidate genes by stepwise exclusion of the worst scoring gene and repeated recalculation of the average $M$ value. Unlike geNorm and BestKeeper, NormFinder employs a modelbased approach, which does not only estimate the overall variation of the candidate genes but also the variation between sample subgroups. All analyses were done correcting for different amplification efficiencies. $C_{q}$ values were transformed into relative quantities for data processing by geNorm and NormFinder using the comparative $C_{\mathrm{q}}$ method and $E$ as base [18]. To assess the expression stability of candidate reference genes in paired samples of unstimulated and stimulated cells, and to evaluate the impact of different normalization strategies on target gene expression, relative expression ratios $(R)$ were calculated for reference genes, combinations of reference genes and target genes using the equation $R=$ $E^{\Delta \mathrm{Cq}}$ where $E$ is the efficiency of the respective real-time PCR assay and $\Delta C_{\mathrm{q}}=\mathrm{C}_{\mathrm{q}}$ (stimulated sample) $-\mathrm{C}_{\mathrm{q}}$ (unstimulated control). These ratios or the geometric means, respectively, were used for calculation of normalized relative expression ratios as described by Pfaffl et al. [19]. Differences in target gene expression were tested for statistical significance $(\mathrm{p}<0.05)$ using paired t-test and Bonferroni correction to account for multiple comparisons.

\section{Results}

Raw $\mathrm{C}_{\mathrm{q}}$ values are summarized in Additional File 2 Table S2. Candidate reference genes were evaluated in a stepwise procedure: First, 17 commonly used reference genes were evaluated in unstimulated and stimulated $\mathrm{T}$ cells. Second, candidate genes stably expressed in T cells were further evaluated in unstimulated and stimulated neutrophils. Finally, candidate reference genes stably expressed in both $\mathrm{T}$ cells and neutrophils were analyzed in total blood leukocytes in order to identify universal leukocyte normalizers.

\section{Reference gene evaluation in unstimulated and anti-CD3/ CD28 stimulated T cells}

The expression of 17 commonly used reference genes (Table 1) was measured by RT-qPCR in paired samples $(n=6)$ of unstimulated and anti-CD3/CD28 stimulated 
$\mathrm{T}$ cells. Attention was paid to selecting candidate genes whose proteins belong to different functional classes to reduce the risk of coregulation. Particularly for valid NormFinder analysis, it is important that the candidates are chosen from a set of genes with no prior expectation of expression differences between subgroups [4]. Genes that differed significantly in their $\mathrm{C}_{\mathrm{q}}$ values between unstimulated and stimulated $\mathrm{T}$ cells (paired t test, $\mathrm{p}<$ 0.05; HPRT1, HMBS, PGK1, PPIA, ACTB, RPLPO, B2M, $A L A S, T F R C, Y W H A Z$ ), and thus had a high chance of being differentially regulated upon stimulation, were therefore excluded from further analysis (Figure 1A), leaving a set of seven candidate genes (18S, HBB, IPO8, RPL13A, SDHA, TBP, GAPDH). The observed $\mathrm{C}_{\mathrm{q}}$ values were distributed over a wide range, including highly expressed $\left(18 S, \mathrm{C}_{\mathrm{q}} \pm \mathrm{SD}, 10.4 \pm 0.6\right)$ as well as far less transcribed genes $(H B B, 29.0 \pm 0.9)$, which violated the assumption of equal variances as a prerequisite for valid
Pearson correlation analysis [5]. We therefore restricted BestKeeper analysis to $\mathrm{C}_{\mathrm{q}}$ variation analysis. RPL13A, TBP and IPO8 showed the lowest standard deviations (Table 2) and were thus considered the most stable reference genes according to BestKeeper. In good agreement, they were also listed among the three or four most stable genes by NormFinder and geNorm, respectively (see Table 3), whereas GAPDH and $H B B$ were consistently ranked the least stable candidates by all three programs. The stability values of the geNorm (individual $M$ values for each gene) and NormFinder analyses are given in Table 2.

\section{Reference gene evaluation in unstimulated and LPS-} stimulated neutrophils

It was one aim of our study to identify potential reference genes that could be used to normalize gene expression data in as many leukocyte subtypes as possible.

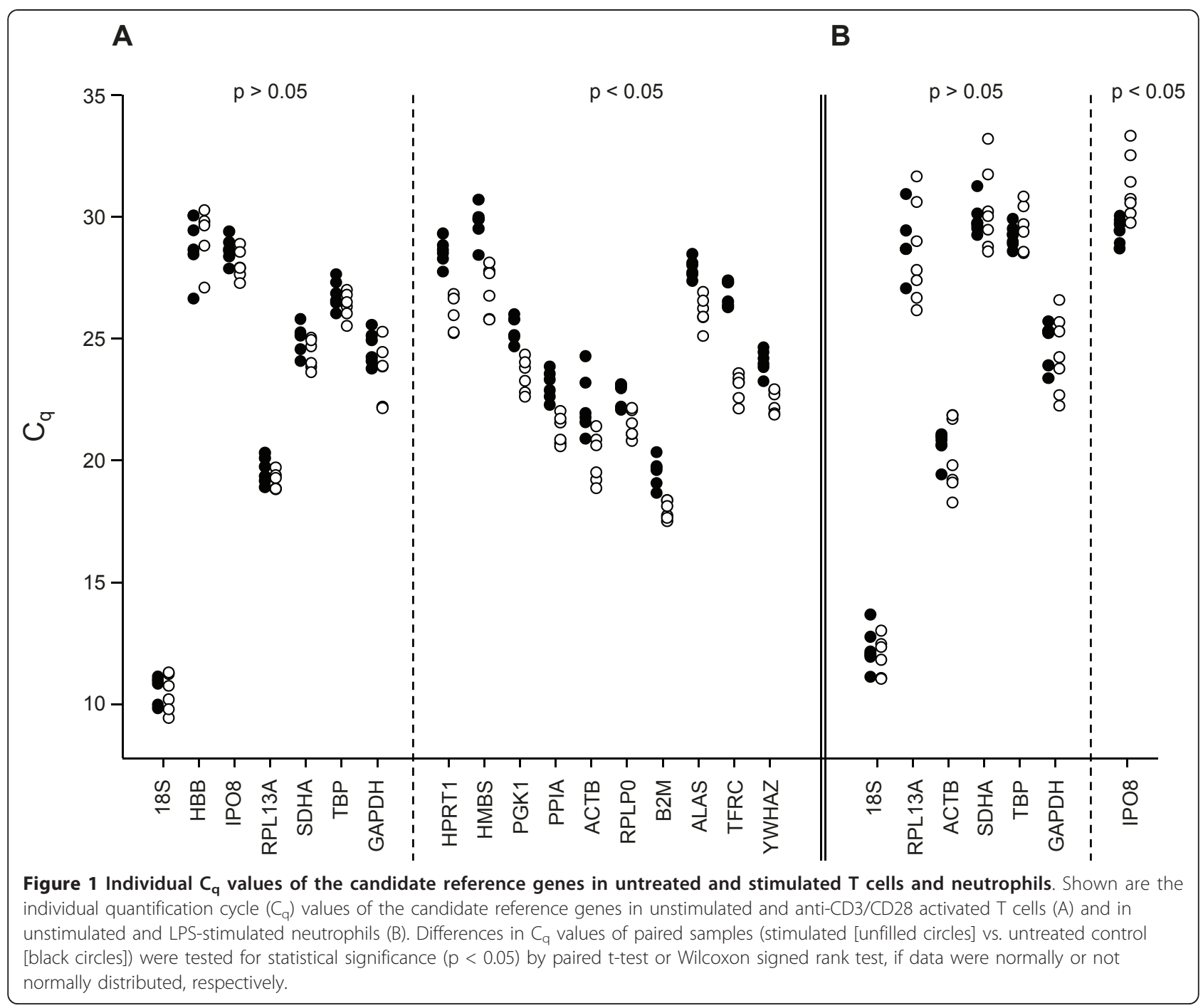


Table 2 Results of BestKeeper, geNorm and NormFinder analyses in unstimulated and anti-CD3/CD28 stimulated T cells

\begin{tabular}{llllllll}
\hline & TBP & IPO8 & SDHA & RPL13A & GAPDH & HBB & 18S \\
\hline GM $\left[C_{q}\right]^{\#}$ & 26.99 & 28.29 & 24.62 & 19.31 & 24.05 & 29.00 & 10.44 \\
AM $\left[C_{q}\right]^{\#}$ & 26.99 & 28.30 & 24.63 & 19.31 & 24.08 & 29.02 & 10.46 \\
$\min \left[C_{q}\right]^{\#}$ & 25.99 & 27.23 & 23.58 & 18.76 & 22.09 & 26.60 & 9.35 \\
$\max \left[C_{q}\right]^{\#}$ & 27.91 & 29.36 & 25.76 & 20.25 & 25.52 & 30.24 & 11.22 \\
SD $\left[ \pm C_{q}\right]^{\#}$ & 0.45 & 0.52 & 0.55 & 0.40 & 0.81 & 0.94 & 0.58 \\
$\mathrm{CV}\left[\% C_{q}\right]^{\#}$ & 1.68 & 1.82 & 2.22 & 2.07 & 3.35 & 3.25 & 5.53 \\
$M$ & 0.941 & 0.873 & 1.035 & 0.793 & 1.203 & 1.508 & 0.920 \\
$S$ & 0.168 & 0.142 & 0.204 & 0.083 & 0.252 & 0.384 & 0.169 \\
\hline
\end{tabular}

Expression stability of potential reference genes was calculated for $n=6$ paired samples of unstimulated and anti-CD3/CD28 activated T cells. $\mathrm{GM}$, geometric mean; $\mathrm{AM}$, arithmetic mean; $\mathrm{C}_{\mathrm{q}}$ quantification cycle; $\mathrm{SD}$, standard deviation; CV, coefficient of variation; "BestKeeper statistics; $M$, stability value determined by geNorm; $S$, stability value determined by NormFinder; expression stability decreases with increasing $M$ and $S$ values.

Table 3 Stability ranking of candidate reference genes in T cells, neutrophils and unselected blood leukocytes by NormFinder, geNorm and BestKeeper

\begin{tabular}{|c|c|c|c|}
\hline & \multicolumn{3}{|c|}{ T cells } \\
\hline Rank & NormFinder & geNorm & BestKeeper \\
\hline 1 & RPL13A & RPL13A/IPO8 & RPL13A \\
\hline 2 & IPO8 & & TBP \\
\hline 3 & TBP & 185 & IPO8 \\
\hline 4 & $18 \mathrm{~S}$ & TBP & SDHA \\
\hline 5 & SDHA & SDHA & 185 \\
\hline 6 & GAPDH & GAPDH & GAPDH \\
\hline \multirow[t]{2}{*}{7} & $\mathrm{HBB}$ & HBB & $\mathrm{HBB}$ \\
\hline & \multicolumn{3}{|c|}{ Neutrophils } \\
\hline Rank & NormFinder & geNorm & BestKeeper \\
\hline 1 & ACTB & SDHA/RPL13A & TBP \\
\hline 2 & TBP & & $18 \mathrm{~S}$ \\
\hline 3 & $\mathrm{SDHA}$ & TBP & SDHA \\
\hline 4 & GAPDH & ACTB & ACTB \\
\hline 5 & $18 \mathrm{~S}$ & GAPDH & GAPDH \\
\hline \multirow[t]{2}{*}{6} & RPL13A & 185 & RPL13A \\
\hline & \multicolumn{3}{|c|}{ Total Blood Leukocytes } \\
\hline Rank & NormFinder & geNorm & BestKeeper \\
\hline 1 & $\mathrm{SDHA}$ & SDHA/TBP & 185 \\
\hline 2 & TBP & & TBP \\
\hline 3 & IPO8 & $18 \mathrm{~S}$ & SDHA \\
\hline 4 & GAPDH & RPL13A & RPL13A \\
\hline 5 & RPL13A & IPO8 & IPO8 \\
\hline 6 & $18 S$ & GAPDH & GAPDH/ACTB \\
\hline 7 & ACTB & ACTB & \\
\hline 8 & HBB & $\mathrm{HBB}$ & $\mathrm{HBB}$ \\
\hline
\end{tabular}

Ranking is based on the stability values (NormFinder), the average expression stability during stepwise exclusion of the least stable gene (geNorm), or SD \pm $C_{\mathrm{q}}$ (BestKeeper). Rank 1 represents the most stable reference gene or combination of reference genes. GeNorm analysis does not allow the ranking of the two most stable genes because its gene-stability measurements require the use of gene ratios.
Therefore, when selecting potential reference genes for the cell culture model of unstimulated and LPS-treated neutrophils ( $\mathrm{n}=7$ paired samples), we focused on the set of seven pre-selected genes that we had evluated in $\mathrm{T}$ cells, with one slight modification: instead of $H B B$, which had been consistently ranked last in $\mathrm{T}$ cells by all three analyzing programs (Table 3 ), ACTB was included, as it is one of the most commonly used reference genes [6] and has previously been suggested for normalization of gene expression in untreated neutrophils [12]. IPO8 expression differed significantly between unstimulated and stimulated neutrophils (paired $t$ test, Figure 1B), and $I P O 8$ was therefore excluded from further analysis, which was finally restricted to $18 S, R P L 13 A, S D H A$, $T B P, G A P D H$ and $A C T B$. As compared to the results obtained in $\mathrm{T}$ cells, the ranking of the candidate genes in neutrophils differed slightly more between the three programs (Table 3). However, ACTB, TBP and SDHA were consistently ranked among the three (NormFinder) or four (geNorm, BestKeeper) most stable genes. The BestKeeper statistics and NormFinder and geNorm stability values are given in Table 4 .

\section{Reference gene evaluation in total blood leukocytes}

Given that neutrophils and $\mathrm{T}$ cells together represent more than $80 \%$ of peripheral blood leukocytes, genes that proved to be suitable for normalization of gene expression in $\mathrm{T}$ cells as well as neutrophils should be promising "universal normalizer" candidates in unselected leukocytes. To test this hypothesis, we assessed the expression stability of the pre-selected candidate genes (TBP, ACTB, SDHA, 18S, RPL13A, HBB, GAPDH, IPO8) in $\mathrm{n}=12$ samples of untreated total blood leukocytes from healthy volunteers (Table 5). In good agreement with the results obtained separately for the leukocyte subtypes, SDHA and TBP were ranked among the two or three best candidates by all three programs,

Table 4 Results of BestKeeper, geNorm and NormFinder analyses in unstimulated and LPS-stimulated neutrophils

\begin{tabular}{lllllll}
\hline & TBP & SDHA & 18S & RPL13A & GAPDH & ACTB \\
\hline $\mathrm{GM}\left[\mathrm{C}_{q}\right]^{\#}$ & 29.27 & 30.03 & 11.94 & 28.60 & 24.54 & 20.37 \\
$\mathrm{AM}\left[\mathrm{C}_{q}\right]^{\#}$ & 29.28 & 30.06 & 11.96 & 28.64 & 24.57 & 20.40 \\
$\min \left[\mathrm{C}_{q}\right]^{\#}$ & 28.47 & 28.54 & 10.96 & 26.12 & 22.20 & 18.21 \\
$\max \left[\mathrm{C}_{q}\right]^{\#}$ & 30.80 & 33.17 & 13.60 & 31.63 & 26.54 & 21.80 \\
$\mathrm{SD}\left[ \pm \mathrm{C}_{q}\right]^{\#}$ & 0.56 & 0.87 & 0.60 & 1.19 & 1.07 & 0.92 \\
$\mathrm{CV}\left[\% \mathrm{C}_{q}\right]^{\#}$ & 1.90 & 2.90 & 5.05 & 4.14 & 4.35 & 4.53 \\
$\mathrm{M}$ & 0.810 & 0.879 & 0.969 & 0.968 & 0.935 & 0.800 \\
$\mathrm{~S}$ & 0.170 & 0.204 & 0.229 & 0.230 & 0.218 & 0.155 \\
\hline
\end{tabular}

Expression stability of potential reference genes was calculated for $n=7$ paired samples of unstimulated and LPS-stimulated neutrophils.

$\mathrm{GM}$, geometric mean; $\mathrm{AM}$, arithmetic mean; $\mathrm{C}_{\mathrm{q}}$, quantification cycle; SD, standard deviation; CV, coefficient of variation; "BestKeeper statistics; $M$, stability value determined by geNorm; $S$, stability value determined by NormFinder; expression stability decreases with increasing $M$ and $S$ values. 
Table 5 Results of BestKeeper, geNorm and NormFinder analyses in total blood leukocytes $(n=12)$

\begin{tabular}{|c|c|c|c|c|c|c|c|c|}
\hline & TBP & IPO8 & SDHA & RPL13A & GAPDH & HBB & ACTB & $18 \mathrm{~S}$ \\
\hline$\overline{\mathrm{GM}\left[\mathrm{C}_{\mathrm{q}}\right]^{\#}}$ & 28.45 & 29.33 & 27.84 & 20.39 & 23.23 & 17.55 & 20.34 & 11.17 \\
\hline $\mathrm{AM}\left[\mathrm{C}_{\mathrm{q}}\right]^{\#}$ & 28.46 & 29.36 & 27.86 & 20.41 & 23.26 & 17.62 & 20.38 & 11.19 \\
\hline $\min \left[C_{q}\right]^{\#}$ & 27.06 & 27.06 & 26.08 & 19.05 & 20.60 & 15.23 & 18.21 & 10.17 \\
\hline $\max \left[C_{q}\right]^{\#}$ & 30.53 & 31.30 & 29.62 & 22.54 & 26.12 & 20.86 & 21.80 & 12.31 \\
\hline $\mathrm{SD}\left[ \pm \mathrm{C}_{\mathrm{q}}\right]^{\#}$ & 0.66 & 0.85 & 0.67 & 0.72 & 1.01 & 1.24 & 1.01 & 0.45 \\
\hline $\mathrm{CV}\left[\% \mathrm{C}_{\mathrm{q}}\right]^{\#}$ & 2.33 & 2.90 & 2.40 & 3.51 & 4.32 & 7.03 & 4.98 & 3.98 \\
\hline M & 0.555 & 0.629 & 0.550 & 0.717 & 0.720 & 1.178 & 0.719 & 0.668 \\
\hline S & 0.173 & 0.322 & 0.145 & 0.378 & 0.333 & 0.781 & 0.407 & 0.387 \\
\hline
\end{tabular}

GM, geometric mean; $A M$, arithmetic mean; $C_{q}$, quantification cycle; SD, standard deviation; $C V$, coefficient of variation; ${ }^{\#} B e s t K e e p e r$ statistics; $M$, stability value determined by geNorm; $S$, stability value determined by NormFinder; expression stability decreases with increasing $M$ and $S$ values.

as opposed to $H B B, A C T B$ and $G A P D H$, which had before turned out to be less stably expressed in $\mathrm{T}$ cells and/or neutrophils. Table 3 summarizes the ranking of the respective candidate genes in $\mathrm{T}$ cells, neutrophils and total blood leukocytes according to the three different analyzing tools.

\section{Optimal number of reference genes}

Normalization by using a normalization factor (NF) based on multiple reference genes rather than a single gene is likely to provide more robust and reliable results [6]. To assess the optimal number of reference genes, geNorm calculates the pairwise variations $V_{n} / V_{n+1}$ between two sequential NFs to determine the effect of adding the next stable reference gene to the NF. As shown in Figure 2, using more than two reference genes would not reduce variation in T cells. Similarly, normalizing to two reference genes would be sufficient in total blood leukocytes showing very low variation values well below the arbitrary threshold of 0.15 . In contrast, adding up to six reference genes led to further reduction in variation in neutrophils. We used NormFinder to corroborate the results. NormFinder calculates stability values $S$ for each candidate gene and the best combination of two genes based on intra- and intergroup variation. After the selection of suited genes based on the estimated intergroup expression variation, the intragroup variance estimates can be used to determine the number of reference genes to include into the NF. The optimal number is reached when addition of a further gene leads to a negligible reduction in the average of gene variance estimates [4]. In T cells using a NF including

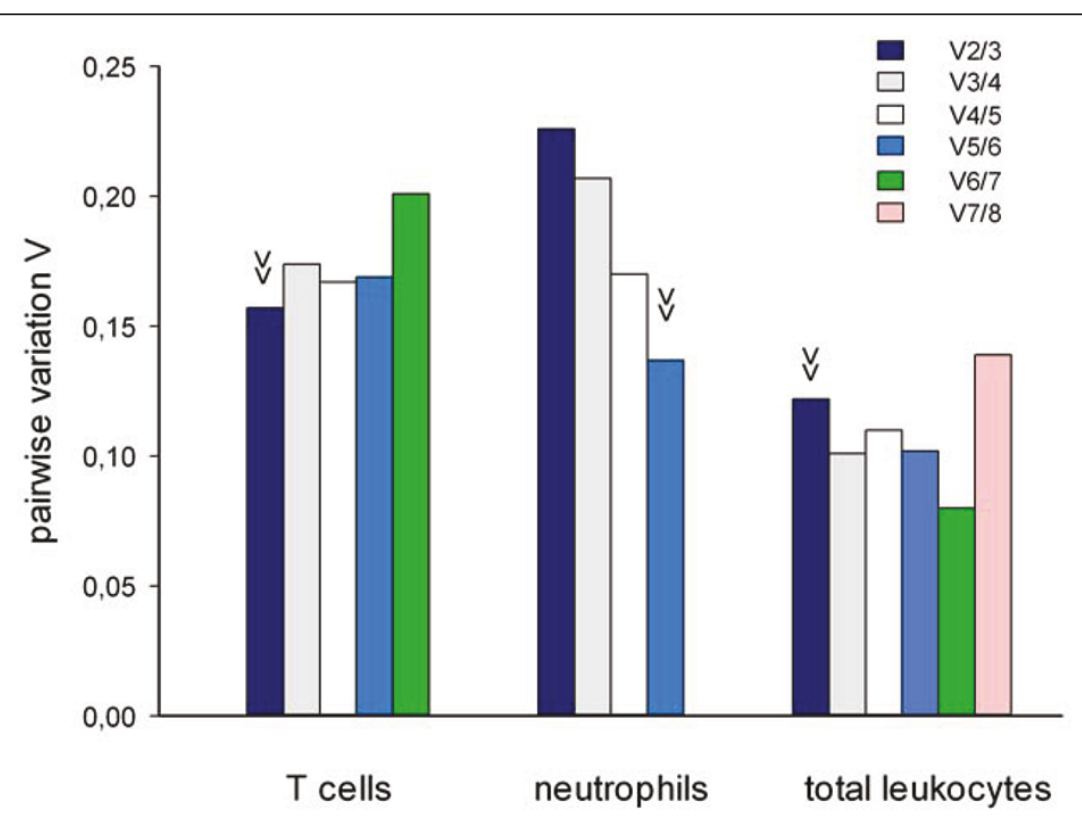

Figure 2 Determination of the optimal number of reference genes using geNorm. Pair-wise variation $\left(V_{n} / n+1\right)$ analysis between the normalization factors $\mathrm{NF}_{\mathrm{n}}$ and $\mathrm{NF}_{\mathrm{n}+1}$ to determine the number of control genes required for normalization was performed (arrowhead indicates optimal number). For ranking order of candidate reference genes see Table 3, geNorm ranking. 
RPL13A and IPO8 led to a reduction in $S$ (0.057 vs. $0.083)$ and the avarage of intragroup variance estimates ( $V_{\text {intra }} ; 0.019$ vs. 0.046 ) as compared to using RPL13A alone. Addition of $T B P$ to the NF did not further improve results $\left(S=0.120, V_{\text {intra }}=0.055\right)$. In neutrophils the combination of $A C T B$ and TBP $\left(S=0.061 ; V_{i n-}\right.$ tra $=0.019)$ performed better than $A C T B$ alone $(S=$ $\left.0.155 ; V_{\text {intra }}=0.099\right)$. A NF including $A C T B, T B P$ and $S D H A$ meant no improvement $\left(S=0.086 ; V_{\text {intra }}=\right.$ 0.052). In the single group of total blood leukocytes inter- and intragroup variance estimates were not calculated. Based on the $S$ values, including multiple reference genes into a NF was not superior to using $S D H A$ alone (SDHA: 0.145; SDHA + TBP: 0.167).

\section{Regulation of reference gene expression in T cells and neutrophils upon stimulation}

Gene expression regulation of unstable reference genes during stimulation will directly influence the estimation of target gene expression. None of the above mentioned programs uses an algorithm that specifically considers paired samples. We therefore validated our results by assessing the expression stability of single candidate reference genes or selected combinations in our experimental setting of paired samples of unstimulated and stimulated cells (Figure 3). Taking into account that most authors recommend the use of multiple reference genes to minimize variation [3-6], we normalized our data to the geometric mean of the three best-performing candidate genes according to the results of all three programs (RPL13A/IPO8/TBP in T cells, ACTB/TBP/SDHA in neutrophils) as an attempt to use the presumably optimal normalization strategy. Overall, the results of the statistical analyses were supported, with the topranking genes in T cells (RPL13A, IPO8, TBP, 18S, $S D H A)$ showing little regulation upon stimulation. In neutrophils slightly higher expression changes were seen. These, however, clearly remained below a 2 -fold change in the top-ranking genes $(A C T B, T B P, S D H A$, $R P L 13 A)$. The extent of expression variation tended to further decrease when analyzing the combined expression of two reference genes. As expected, the candidate genes that had been ruled out from the beginning due to significant intergroup differences in $\mathrm{C}_{\mathrm{q}}$ values displayed the highest variation, with most of them being regulated more than 2 -fold.

\section{Influence of the normalization strategy on the estimated target gene expression}

In order to evaluate the impact of different normalization strategies, we determined the relative change in the expression of two target genes, $I L-2$ and $F I H$, in antiCD3/CD28 stimulated T cells $(n=4)$. We applied three different normalization approaches: (i) normalizing to the geometric mean of IPO8 and RPL13A, the best combination of two genes according to NormFinder and geNorm; (ii) normalizing to $H B B$ or $H P R T 1$, both of which are candidate reference genes frequently used for normalization of RT-qPCR data and had performed poorly in our analyses; (iii) normalizing to the geometric mean of top ranking RPL13A and HBB or HPRT1. As expected, $I L-2$ mRNA levels strongly increased in activated $\mathrm{T}$ cells, and this up-regulation was significant with all tested normalization strategies (Figure 4A), though considerably varying in its extent. Assuming that normalizing to IPO8 and RPL13A provided the most reliable results, using $H P R T 1$ led to a 3.6-fold underestimation and using $H B B$ to a 2.3 -fold overestimation of the expression change. While the tendency, i. e. up-regulation, of $I L-2$ expression was observed irrespectively of the normalization approach, this was not the case when analyzing $F I H$ expression (Figure 4B): while no significant change in gene expression could be detected when using the recommended reference gene combination of RPL13A and IPO8, normalization strategies involving $H P R T$ or $H B B$ resulted in a significant down- or up-regulation, respectively. There is general consensus that normalizing to a single reference gene should be avoided [6]. In agreement, using the combination of a stable and an unstable reference gene for normalization indeed reduced the distorting effect, however the differences in gene expression remained significant.

\section{Discussion}

Quantitative real-time PCR has become a standard method for gene expression analysis, allowing accurate quantification of mRNA levels over a wide dynamic range [2]. If handled improperly, however, the results can be misleading. One of the most critical points is the selection of appropriate reference genes to control for experimental error between samples [3,7]. In the current study, we evaluated, to our knowledge for the first time, the expression stability of common reference genes separately in two widely-used cell culture models of stimulated leukocyte subtypes: $\mathrm{T}$ cells activated by antiCD3/CD28 beads, and LPS-stimulated neutrophils. A major finding of our study was that several conventional "housekeeping genes" proved to be unreliable controls, which is in line with previous reports about an unstable expression of commonly used reference genes, such as GAPDH, $A C T B$ or HPRT1, in various experimental setups [11,20-22]. Of note, IPO8 and $A C T B$ behaved considerably differently regarding their stability in neutrophils or $\mathrm{T}$ cells, and candidate genes we found inappropriate for normalization in activated $\mathrm{T}$ cells have been reported to be stably expressed in LPS-treated monocytes (B2M, PPIA, ACTB [11]) or B cells from chronic lymphocytic leukemia patients (B2M, HPRT1 


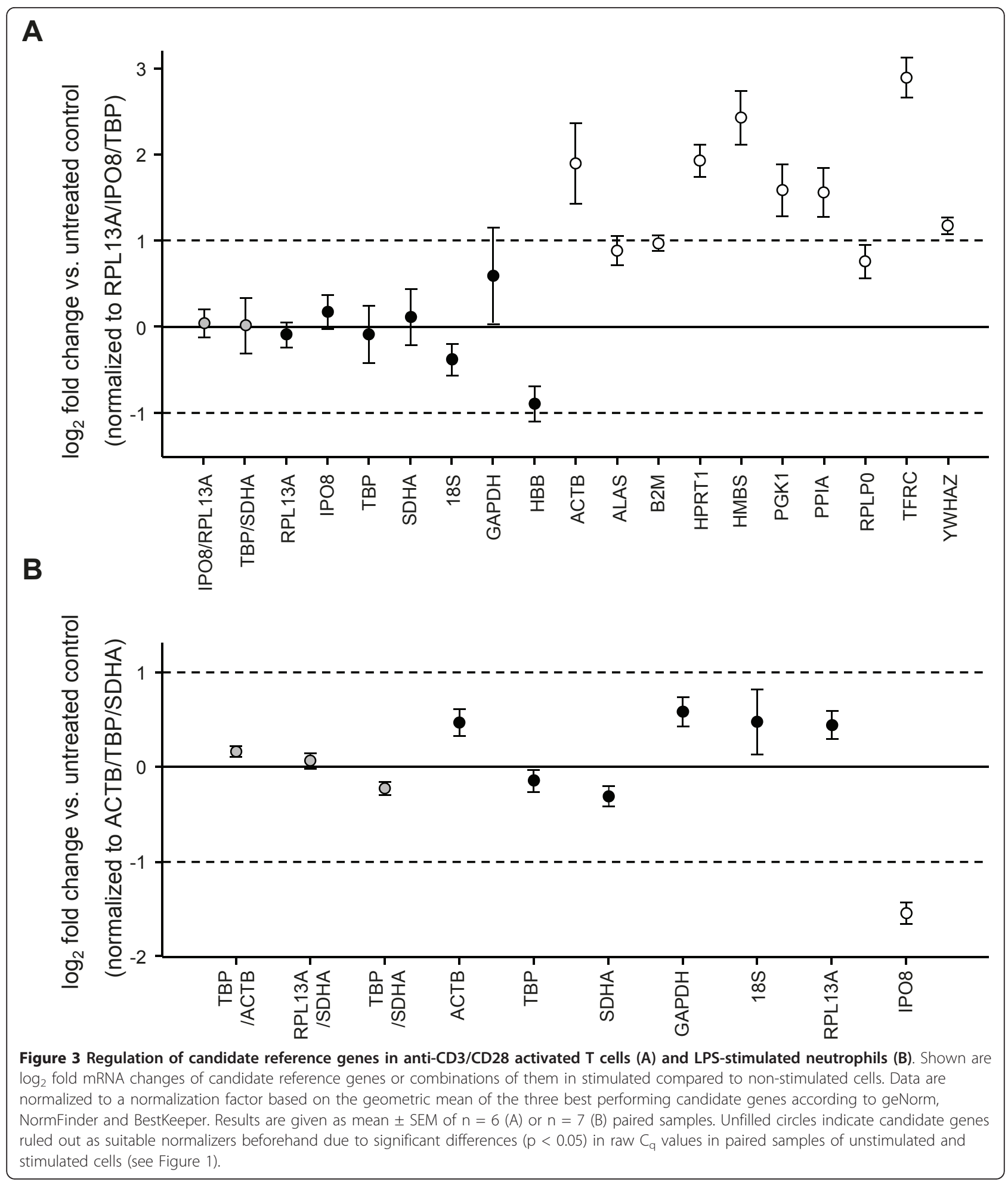

[23]). These findings underscore the necessity of careful individual validation of reference genes for every leukocyte subtype and every experimental condition.

BestKeeper, geNorm and NormFinder outputs provided very similar stability rankings of the candidate genes, especially in $\mathrm{T}$ cells. As the programs are based on different algorithms [4-6], the consensus between them increases the reliability of the results. In neutrophils, there was some discrepancy in the ranking order: geNorm identified RPL13A as one of the two most 


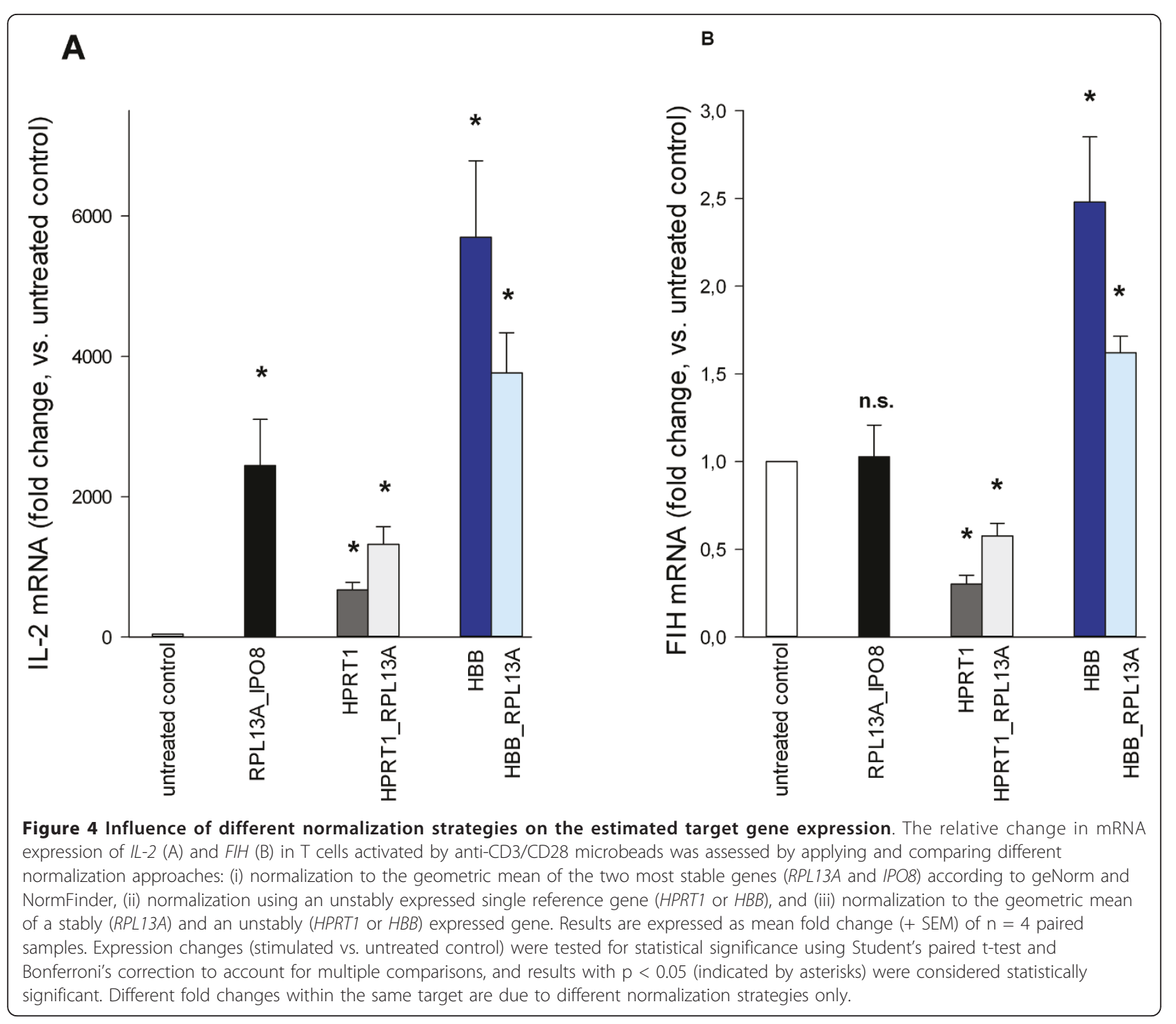

stable genes, whereas RPL13A was assigned the last rank by NormFinder and BestKeeper analyses. In contrast to NormFinder, the pairwise comparison approach applied by geNorm is sensitive to co-regulation and shows a tendency to top rank candidates with correlated expression rather than minimal variation [4], which could be an explanation for differing results. In the present study, the combinations of the two most suitable genes proposed by geNorm (SDHA/RPL13A) and NormFinder $(A C T B / T B P)$ showed a similarly low expression variation in paired samples of untreated and stimulated neutrophils, suggesting the suitability of both normalization approaches. Consistent with the recently published MIQE (minimum information for publication of quantitative real-time PCR experiments) guidelines [1], these results support the use of a normalization strategy that is based on several stably expressed genes, not just a single gene, to reduce variation. The number of reference genes used in a particular experiment will be a compromise between minimizing variability and considerations of practicability $[4,6]$. NormFinder and geNorm consistently suggested the use of two reference genes (RPL13A and IPO8) for normalizing gene expression data in unstimulated and activated T cells. In neutrophils, results differed between geNorm and NormFinder with geNorm indicating the optimal number of reference genes with six, whereas according to NormFinder the combination of $A C T B$ and $T B P$ was sufficient. It is important to note that neither geNorm nor NormFinder claim absolutness of their results but recommend them as a guideline which has to be interpreted individually when selecting the number of reference genes to be used $[4,6]$. Based on the results in paired samples, and considering that NormFinder, unlike geNorm, takes 
intergroup differences into account and is less susceptibel to co-regulation of genes, we recommend the use of at least two genes out of $A C T B, T B P, S D H A$ and RPL13A for normalization in LPS-stimulated neutrophils. $18 S$, which is commonly used for normalization of qPCR data in various cell types [24], including leukocytes $[25,26]$, belonged to the stably expressed candidates in T cells. Due to its high expression, though, it will likely be inappropriate for the expression normalization of most genes of interest, as similar abundances of target and reference gene are important to ensure that they are both subject to the same PCR kinetics [6].

We intended to identify potential "universal leukocyte normalizers" (suitable for as many leukocyte subtypes as possible). Therefore, we limited the reference genes evaluated in neutrophils to those candidates that had performed well in $\mathrm{T}$ cells. As a consequence of this sequential procedure, it cannot be excluded that a subset of reference genes not tested in our study would be suitable for normalizing gene expression in neutrophils. Studying gene expression in total blood leukocytes, thereby circumventing the time-consuming purification of single leukocyte subtypes, appears as an attractive approach in the search for diagnostic or therapeutic targets in immune-mediated disease [9], although one has to be aware of its inherent limitations: changes in expression levels may not only be due to regulation of transcriptional activity but also reflect relative changes in the abundance of single cell populations with constant expression levels. The bias introduced will be especially pronounced if the control genes used for normalization show variable expression stabilities in different leukocyte subtypes. The expression stability of potential reference genes should therefore ideally be assessed in the single cell types prior to using them in mixed-cell approaches. Our results identified the combination of SDHA and TBP as a suitable normalizer in T cells as well as in neutrophils. In good agreement, a recent study recommends the use of SDHA as a reference gene in LPS stimulated porcine T cells [27]. Furthermore, TBP has recently been reported to be stably expressed in LPS stimulated monocytes [11]. We therefore hypothesized that TBP and SDHA could be suitable "universal" reference genes in unselected leukocytes. In support of our results, SDHA and TBP were listed among the three most stable genes in total blood leukocytes by all three analyzing softwares. Although NormFinder analyses found the use of a single reference gene (SDHA) to be sufficient in total blood leukocytes, we recommend as a general rule the use of at least two reference genes, and thus normalization to $S D H A$ and $T B P$, as suggested by geNorm.

Whether a chosen normalization strategy is considered suitable or not in a given experimental setting also depends on the extent and required resolution of expression differences. When analyzing the expression of $I L-2$, a target gene that undergoes a strong upregulation in activated $\mathrm{T}$ cells, even the use of considerably instable reference genes correctly indicated an increase in $I L-2$ transcripts, which may be sufficient if only an on-/off response is to be detected. Usually, however, the investigated regulatory effect is much smaller, and estimating the exact expression change is important. In this case, the use of inappropriate reference genes leads to unreliable results and may even produce artificial changes, as is demonstrated by the comparison of different normalization approaches for the expression of $F I H$, a key component of the cellular oxygen-sensing machinery that controls the activity of the transcriptional regulator HIF-1 $\alpha$ [28], but is not known to be regulated in $\mathrm{T}$ cells activated by anti-CD3/CD28 beads under normoxic conditions. Of note, adding a stable reference gene for normalization did considerably compensate for the distorting effect of using a single unstable reference gene, thus supporting the use of more than one reference gene [6]. However, even when combined with the most stable gene, using an unstably expressed gene led to erronous $F I H$ expression results; a careful selection of all the reference genes used for normalization is therefore required.

\section{Conclusions}

Our study clearly demonstrates the need to carefully select appropriate reference genes for normalization of gene expression data obtained by RT-qPCR. We recommend the use of two genes out of RPL13A, IPO8, TBP and $S D H A$ and at least two genes out of $A C T B, T B P$, $S D H A$ and RPL13A as RT-qPCR control genes in T cells and neutrophils, respectively. Furthermore, $S D H A$ and $T B P$ were shown to be suitable gene expression normalizers in unselected leukocytes.

\section{Availability of supporting data}

The data sets supporting the results of this article are included within the article and its additional files.

\section{Additional material}

Additional file 1: Table S1-Real-time PCR assay characteristics. This table summarizes the characteristics of the GPCR assays used in this study, including assay ID, amplicon start and end point, amplification efficiency $E$ and $r^{2}$.

Additional file 2: Table $S 2-C_{q}$ values of candidate reference genes. Single $C_{q}$ values of all candidate reference genes evaluated in this study in T cells, neutrophils and total blood leukocytes are listed.

Acknowledgements

We thank J. Rink for her excellent technical assistance. 


\section{Author details}

'Department of Anesthesiology and Intensive Care Medicine, Clinical Faculty Mannheim, University of Heidelberg, Germany. ${ }^{2}$ Department of

Anesthesiology, Ludwig-Maximilians-University Munich, Germany.

\section{Authors' contributions}

$\mathrm{CL}$ designed and performed the experiments, analyzed the data, performed the statistical analysis and wrote the paper. $\mathrm{JH}$ and EL participtated in performing experiments and discussing results. SK concieved of the study, participated in its design and helped to draft the manuscript. All authors read and approved the final manuscript.

\section{Competing interests}

The authors declare that they have no competing interests.

Received: 2 August 2011 Accepted: 20 October 2011

Published: 20 October 2011

\section{References}

1. Bustin SA, Benes V, Garson JA, Hellemans J, Huggett J, Kubista M, Mueller R, Nolan T, Pfaffl MW, Shipley GL, et al: The MIQE guidelines: minimum information for publication of quantitative real-time PCR experiments. Clin Chem 2009, 55(4):611-622.

2. Bustin SA, Benes V, Nolan T, Pfaffl MW: Quantitative real-time RT-PCR-a perspective. J Mol Endocrinol 2005, 34(3):597-601.

3. Thellin $\mathrm{O}$, ElMoualij $\mathrm{B}$, Heinen $\mathrm{E}$, Zorzi W: A decade of improvements in quantification of gene expression and internal standard selection. Biotechnol Adv 2009, 27(4):323-333.

4. Andersen $C L$, Jensen $J$, Orntoft TF: Normalization of real-time quantitative reverse transcription-PCR data: a model-based variance estimation approach to identify genes suited for normalization, applied to bladder and colon cancer data sets. Cancer Res 2004, 64(15):5245-5250.

5. Pfaffl MW, Tichopad A, Prgomet C, Neuvians TP: Determination of stable housekeeping genes, differentially regulated target genes and sample integrity: BestKeeper-Excel-based tool using pair-wise correlations. Biotechnol Lett 2004, 26(6):509-515.

6. Vandesompele J, De Preter K, Pattyn F, Poppe B, Van Roy N, De Paepe A, Speleman F: Accurate normalization of real-time quantitative RT-PCR data by geometric averaging of multiple internal control genes. Genome Biol 2002, 3(7):RESEARCH0034.

7. Huggett J, Dheda K, Bustin S, Zumla A: Real-time RT-PCR normalisation; strategies and considerations. Genes Immun 2005, 6(4):279-284.

8. Thellin O, Zorzi W, Lakaye B, De Borman B, Coumans B, Hennen G, Grisar T, Igout $A$, Heinen $E$ : Housekeeping genes as internal standards: use and limits. J Biotechnol 1999, 75(2-3):291-295.

9. Chaussabel D, Pascual V, Banchereau J: Assessing the human immune system through blood transcriptomics. BMC Biol 2010, 8:84.

10. Maess MB, Sendelbach S, Lorkowski S: Selection of reliable reference genes during THP-1 monocyte differentiation into macrophages. BMC Mol Biol 2010, 11:90.

11. Piehler AP, Grimholt RM, Ovstebo R, Berg JP: Gene expression results in lipopolysaccharide-stimulated monocytes depend significantly on the choice of reference genes. BMC Immunol 2010, 11:21.

12. Zhang $X$, Ding $L$, Sandford AJ: Selection of reference genes for gene expression studies in human neutrophils by real-time PCR. BMC Mol Biol 2005, 6(1):4.

13. Trickett A, Kwan YL: T cell stimulation and expansion using anti-CD3/ CD28 beads. J Immunol Methods 2003, 275(1-2):251-255.

14. Malcolm KC, Arndt PG, Manos EJ, Jones DA, Worthen GS: Microarray analysis of lipopolysaccharide-treated human neutrophils. Am J Physiol Lung Cell Mol Physiol 2003, 284(4):L663-670.

15. Tsukahara $Y$, Lian Z, Zhang X, Whitney C, Kluger Y, Tuck D, Yamaga S, Nakayama Y, Weissman SM, Newburger PE: Gene expression in human neutrophils during activation and priming by bacterial lipopolysaccharide. J Cell Biochem 2003, 89(4):848-861.

16. Zhang $X$, Kluger $Y$, Nakayama $Y$, Poddar R, Whitney C, DeTora A, Weissman SM, Newburger PE: Gene expression in mature neutrophils: early responses to inflammatory stimuli. J Leukoc Biol 2004, 75(2):358-372.

17. Kreth S, Ledderose C, Kaufmann I, Groeger G, Thiel M: Differential expression of $5^{\prime}$-UTR splice variants of the adenosine A2A receptor gene in human granulocytes: identification, characterization, and functional impact on activation. FASEB J 2008, 22(9):3276-3286.

18. Livak KJ, Schmittgen TD: Analysis of relative gene expression data using real-time quantitative PCR and the 2(-Delta Delta $C(T))$ Method. Methods 2001, 25:(4):402-408

19. Pfaffl MW: A new mathematical model for relative quantification in realtime RT-PCR. Nucleic Acids Res 2001, 29(9):e45.

20. Kreth S, Heyn J, Grau S, Kretzschmar HA, Egensperger R, Kreth FW: Identification of valid endogenous control genes for determining gene expression in human glioma. Neuro Oncol 2010, 12(6):570-579.

21. Pilbrow AP, Ellmers LJ, Black MA, Moravec CS, Sweet WE, Troughton RW, Richards AM, Frampton CM, Cameron VA: Genomic selection of reference genes for real-time PCR in human myocardium. BMC Med Genomics 2008, 1:64.

22. Watson S, Mercier S, Bye C, Wilkinson J, Cunningham AL, Harman AN: Determination of suitable housekeeping genes for normalisation of quantitative real time PCR analysis of cells infected with human immunodeficiency virus and herpes viruses. Virol J 2007, 4:130.

23. Valceckiene V, Kontenyte R, Jakubauskas A, Griskevicius L: Selection of reference genes for quantitative polymerase chain reaction studies in purified B cells from B cell chronic lymphocytic leukaemia patients. $\mathrm{Br} J$ Haematol 2010, 151(3):232-238.

24. Goidin D, Mamessier A, Staquet MJ, Schmitt D, Berthier-Vergnes O: Ribosomal 18S RNA prevails over glyceraldehyde-3-phosphate dehydrogenase and beta-actin genes as internal standard for quantitative comparison of mRNA levels in invasive and noninvasive human melanoma cell subpopulations. Anal Biochem 2001, 295(1):17-21.

25. Baines KJ, Simpson JL, Gibson PG: Innate immune responses are increased in chronic obstructive pulmonary disease. PLoS One 2011, 6(3):e18426.

26. Ear T, Fortin CF, Simard FA, McDonald PP: Constitutive association of TGFbeta-activated kinase 1 with the IkappaB kinase complex in the nucleus and cytoplasm of human neutrophils and its impact on downstream processes. J Immunol 2010, 184(7):3897-3906.

27. Facci MR, Auray G, Meurens F, Buchanan R, van Kessel J, Gerdts V: Stability of expression of reference genes in porcine peripheral blood mononuclear and dendritic cells. Vet Immunol Immunopathol 2011, 141(12):11-15.

28. Kaelin WG Jr, Ratcliffe PJ: Oxygen sensing by metazoans: the central role of the HIF hydroxylase pathway. Mol Cell 2008, 30(4):393-402.

doi:10.1186/1756-0500-4-427

Cite this article as: Ledderose et al: Selection of reliable reference genes for quantitative real-time PCR in human T cells and neutrophils. BMC Research Notes 2011 4:427.

\section{Submit your next manuscript to BioMed Central and take full advantage of:}

- Convenient online submission

- Thorough peer review

- No space constraints or color figure charges

- Immediate publication on acceptance

- Inclusion in PubMed, CAS, Scopus and Google Scholar

- Research which is freely available for redistribution

Submit your manuscript at www.biomedcentral.com/submit
C Biomed Central 